\title{
Depression was associated with increased risk of coronary heart disease and death in older adults
}

\author{
Ariyo AA, Haan M, Tangen CM, et al, for the Cardiovascular Health Study Collaborative Research Group. Depressive \\ symptoms and risks of coronary heart disease and mortality in elderly Americans. Circulation 2000 Oct 10;102:1773-9.
}

\section{QUESTION: In older adults, is depression a risk factor for coronary heart disease $(\mathrm{CHD})$ and death?}

Design

6 year cohort study (Cardiovascular Health Study [CHS]).

Setting

4 US communities.

\section{Participants}

4493 participants who were $65-98$ years of age (mean age 72 y, 61\% women) and had no cardiovascular disease were recruited from medical eligibility lists from each county.

\section{Assessment of risk factors}

Depression was assessed at baseline and annually thereafter using the modified, shorter version of the Center for Epidemiological Studies' Depression Scale. Potential confounding variables (age at entry, race, sex, education, hypertension, diabetes, smoking status, total cholesterol concentrations, physical inactivity, triglycerides, marital status, and alcohol consumption) were also assessed.

\section{Main outcome measures}

CHD defined as first occurrence of angina, myocardial infarction, angioplasty, coronary artery bypass grafting, or coronary death; and all cause mortality.

\section{Main results}

The mean depression score was assessed for each participant at the time of a CHD event or death or for up to 6 years of follow up. Every 5 point increase in mean depression score on a $0-30$ point scale was associated with a $15 \%$ increase in risk of developing CHD (unadjusted and multivariate analysis, $\mathrm{p}=0.006$ ). Increases of $16 \%$ (unadjusted, $\mathrm{p}<0.001$ ) and $29 \%$ (multivariate, $\mathrm{p}=0.006$ ) were observed in risk of death. The table gives the adjusted hazard ratios. The findings held equally true for men and women.

\section{Conclusions}

In older adults, depression was an independent risk factor for coronary heart disease and death. The risk increased with higher depression scores.

Source of funding: National Heart, Lung, and Blood Institute.

For correspondence: $\operatorname{Dr}$ A A Ariyo, Cardiovascular Division, Johns Hopkins Hospital, 600 $N$ Wolfe Street, Carnegie 568,

Baltimore, $M D 21287$

USA. Fax +1806780 5423. depression score and coronary heart disease events and death at up to 6 years*

\begin{tabular}{lll} 
Outcomes & $\begin{array}{l}\text { Number of } \\
\text { events }\end{array}$ & $\begin{array}{l}\text { Adjusted hazard } \\
\text { ratio }(95 \% \mathrm{Cl})\end{array}$ \\
\hline Coronary heart disease & 606 & $1.15(1.04$ to 1.27$)$ \\
\hline All cause mortality & 614 & $1.16(1.04$ to 1.28$)$ \\
\hline
\end{tabular}

*Analysis adjusted for age at entry, race, sex, education, hypertension, diabetes, smoking status, total cholesterol concentrations, physical inactivity, diabetes, smoking status, total cholesterol concentrations, physical inactivity,
triglycerides, marital status, and alcohol consumption (for mortality also adjusted for chronic obstructive pulmonary disease and cancer).
Association between 5 unit increases in cumulative mean

\section{COMMENTARY}

The past decade has seen growing evidence of depression as a physical health risk factor in addition to its mental health implications. Researchers are painfully aware that making a causal connection between an alleged risk factor and a long term health outcome is a laborious undertaking. When attempting to make such connections it is imperative that the presence of the risk factor can be shown to have preceded the disease, that a plausible biological pathway exists, and that other risk factors cannot already account for the variance in statistical predictions. It is of particular clinical value when such studies target a risk factor that is actually treatable, and depression certainly is. For these reasons, Ariyo et al's study makes an important contribution to this body of research because it shows that depression predicts a critical endpoint in a large, representative sample. Adjustment for other known demographic, biological, and health behaviour risks does not eliminate or even weaken the strength of the finding. Because this is an epidemiological study, it cannot inform us about a biological pathway, but the consistency with which depression has been found to predict negative health outcomes strongly suggests that the search for pathways is worthwhile and informative for treatment formulations.

The authors deserve praise for a high quality addition to the literature, but it should be noted that this finding is not original; while previous findings are effectively replicated here, no mention is made of the Coronary Artery Risk Development in Young Adults (CARDIA) study which produced similar results. ${ }^{1}$ In CARDIA, depression was found to predict hypertension thus suggesting 1 possible pathway for depression leading to $\mathrm{CHD}$ and overall mortality.

Some researchers might look at the observed hazard ratios (ranging from 1.15 to 1.29 ) and sneer at their relatively small size, but at least 2 reasons argue against this. Firstly, in Ariyo et al's study, participants were followed for 5 years, and the 1.15 hazard ratio applies to each 5 point increment such that the effects of depression and mortality outcomes across the entire scale range are not immediately obvious. Secondly, I think it is remarkable that a simple, inexpensive, 10 item questionnaire is sufficiently sensitive to detect such an effect. Typically, screening instruments have low discriminative power and may underestimate the strength of linkages, and I speculate that a more sophisticated assessment of depression (with its inherent superior reliability) might also result in correspondingly higher risk ratios.

Wolfgang Linden, $\mathrm{PhD}$ University of British Columbia Vancouver, British Columbia, Canada

1 Davidson K, Jonas BS, Dixon KE, et al. Do depression symptoms predict early hypertension incidence in young adults in the CARDIA study? Coronary artery risk development in young adults. Arch Intern Med 2000;160:1495-500. 